\title{
Bohmian quantum mechanics revisited
}

\author{
A. I. ARBAB (a) \\ Department of Physics, College of Science, Qassim University, P.O. Box 6644, 51452 Buraidah, KSA \\ Department of Physics, Faculty of Science, University of Khartoum, Khartoum 11115, Sudan
}

PACS 03.65.-w - Quantum mechanics

PACS 03.65. Ca - Formalism

PACS 03.75.-b-Matter waves

\begin{abstract}
By expressing the Schrödinger wave function in the form $\psi=R e^{i S / \hbar}$, where $R$ and $S$ are real functions, we have shown that the expectation value of $S$ is conserved. The amplitude of the wave $(R)$ is found to satisfy the Schrödinger equation while the phase $(S)$ is related to the energy conservation. Besides the quantum potential that depends on $R$, viz., $V_{Q}=-\frac{\hbar^{2}}{2 m} \frac{\nabla^{2} R}{R}$, we have obtained a spin potential $V_{S}=-\frac{S \nabla^{2} S}{m}$ that depends on $S$ which is attributed to the particle spin. The spin force is found to give rise to dissipative viscous force. The quantum potential may be attributed to the interaction between the two subfields $S$ and $R$ comprising the quantum particle. This results in splitting (creation/annihilation) of these subfields, each having a mass $m c^{2}$ with an internal frequency of $2 m c^{2} / \hbar$, satisfying the original wave equation and endowing the particle its quantum nature. The mass of one subfield reflects the interaction with the other subfield. If in Bohmian ansatz $R$ satisfies the Klein-Gordon equation, then $S$ must satisfies the wave equation. Conversely, if $R$ satisfies the wave equation, then $S$ yields the Einstein relativistic energy momentum equation.
\end{abstract}

KEYWORDS: Quantum Mechanics; Bohmian Quantum Mechanics; Quantum Potential; Schrodinger Equation; Dirac Equation; Klein-Gordon Equation, Spin

Introduction. - To give a conceivable meaning for Schrödinger quantum mechanics, Bohm had expressed the wavefunction representing the particle in the form $\psi=R e^{i S / \hbar}$, where $R$ and $S$ are two real functions [1-3]. In doing so, Bohm had reformulated quantum mechanics by describing the particle classical trajectory by a quantum Hamilton-Jacobi equation while retaining the quantum aspect of the particle. Thus, his formulation includes some hidden variables that also describe the state of the particle. The particle is described by the action $S$ that satisfies the Hamilton-Jacobi equation, and $R$ satisfying the continuity equation. In the framework of this recipe, a classical spin potential is derived whose force yields a viscous force. The coefficient of viscosity is found to be directly proportional to the spin.

In this paper, we follow the same routine as Bohm but define another action-like quantity, that is $\hbar R$, associated with a different Hamiltonian. Inasmuch as the ordinary Hamiltonian encompasses a quantum potential, the new Hamiltonian gives rise to a different quantum potential that we refer to spin potential. In this formula-

(a) arbab.ibrahim@gmail.com 
tion, the wave amplitude $(R)$ is found to satisfy the Schrödinger equation. The solution of this equation yields the spatial dependence of $R$, but the time dependence is obtained by solving the Hamilton equations of motion by treating $S$ and $R$ and their spatial derivatives $(\vec{\nabla} S$ and $\hbar \vec{\nabla} R$ ) as the corresponding generalized momenta. Solving the Hamilton's equations for each system yields the relation between $R$ and $S$. In the Bohm formulation, the functions $R$ and $S$ are arbitrarily related functions. For a particular choice of the generalized co-ordinates $(S=2 \pi \hbar R)$, the quantum potential arising from $R$ curvature is equal to that arising from $S$ curvature.

We apply the Bohm recipe to the Dirac equation, and the quantum telegraph equation that was recently shown to yield quantum mechanical identifications of quantum particles [4]. The amplitude $(R)$ satisfies the original quantum telegraph equation, but the phase $(S)$ satisfies the wave equation traveling at the speed of light. In a particular case, $S$ is shown to satisfy the relativistic Einstein energy-momentum equation if $R$ satisfies a special telegraph equation. A similar situation applies for the Klein-Gordon equation too. We treat here the functions $R$ and $S$, in the Klein-Gordon and the unified wave equations, as fields in which the wavefunction (field) is decomposed. They can also be thought of as eigen functions (basis) for the original wavefunction.

In this paper we present in Section 2 the standard Bohmian mechanics where we derived a new potential besides the quantum potential. In Section 3 we employ the canonical quantization by treating the probability conservation equations as defining another Hamilton-Jacobi equation. We then obtained the equation of motion of the two subfields $R$ and $S$ by treating them as generalized co-ordinates. We explore the Bohmian mechanics in Dirac formalism in Section 5. Section 6 and 7 are dealt with the Bohmian mechanics for the Klein-Gordon and the unified wave equations (the telegraph quantum equation). We end our paper by some concluding remarks.

Bohmian quantum mechanics. - Substituting $\psi=R e^{i S / \hbar}$ in the Schrödinger's equation and equating the real and imaginary parts in the two sides of the resulting equation to each other, yield [1]

$$
\frac{\partial S}{\partial t}=-\left(\frac{(\nabla S)^{2}}{2 m}+V-\frac{\hbar^{2}}{2 m} \frac{\nabla^{2} R}{R}\right)
$$

and

$$
\frac{\partial R}{\partial t}=-\frac{1}{2 m}\left(R \nabla^{2} S+2 \vec{\nabla} R \cdot \vec{\nabla} S\right)
$$

the term $V_{Q}=-\frac{\hbar^{2}}{2 m} \frac{\nabla^{2} R}{R}$ is known as the quantum potential. It represents the quantum effects observed in a system. It also reflects a curvature effect resulting from $R$. The presence of this term in the quantum HamiltonJacobi equation helps to view the quantum effects along a classical trajectory. The quantum potential also acts to guide the movement of the quantum particle. The relation between $R$ and $S$ is obtained by expressing $\psi$ as $\psi=U+i W=R \cos (S / \hbar)+i R \sin (S / \hbar)$. This implies that $R^{2}=U^{2}+W^{2}$ and $S=\hbar \tan ^{-1}(W / U)$. Notice that $U$ and $W$ satisfy the Schrodinger's equation, while $R$ and $S$ do not. 
We would like here to explore the time evolution of the expectation value of $S$, according to quantum mechanics, invoking the fluid concept of the particle. The expectation value of $S$ is defined by $<>>$ $\int \psi^{*} S \psi d \tau=\int R^{2} S d \tau$, where $d \tau$ is the volume element. Owing to Ehrenfest theorem, the expectation of a quantum quantity behaves like its classical analogue. To this aim, we multiply eq.(1) by $R^{2}$ and eq.(2) by $2 R S$ and add the two resulting equations to obtain

$$
\frac{\partial}{\partial t}\left(R^{2} S\right)+\frac{R^{2}(\nabla S)^{2}}{2 m}+R^{2} V-\frac{\hbar^{2}}{2 m} R \nabla^{2} R+\frac{R^{2} S \nabla^{2} S}{m}+\frac{2 R S(\vec{\nabla} R \cdot \vec{\nabla} S)}{m}=0 .
$$

We further know that

$$
\frac{d}{d t}\left(R^{2} S\right)=\frac{\partial\left(R^{2} S\right)}{\partial t}+\vec{v} \cdot \vec{\nabla}\left(R^{2} S\right)
$$

But since $\vec{v}=\frac{\vec{\nabla} S}{m}$, then eq.(4) becomes

$$
\frac{d}{d t}\left(R^{2} S\right)=\frac{\partial}{\partial t}\left(R^{2} S\right)+\frac{\vec{\nabla} S}{m} \cdot \vec{\nabla}\left(R^{2} S\right)
$$

The phase of the wave, which can be chosen to represent the classical spin of the particle, is an internal property, will now have a physical meaning (measurable quantity). If this spin is conserved, then the right hand-side of eq.(5) vanishes, and hence one can write

$$
\frac{\partial}{\partial t}\left(R^{2} S\right)+\frac{2 R S(\vec{\nabla} S \cdot \vec{\nabla} R)}{m}+\frac{R^{2}(\nabla S)^{2}}{m}=0
$$

by expanding the second term in the right hand-side of eq.(5).

Let us now combine eqs.(3) and (6) to obtain

$$
\frac{\hbar^{2}}{2 m} \frac{\nabla^{2} R}{R}-V=\frac{S \nabla^{2} S}{m}-\frac{(\nabla S)^{2}}{2 m} .
$$

Now if we assume that $V$ is a function of $R$ only, one can solve eq.(7) by setting each side equal to a constant, E, viz.

$$
\frac{\hbar^{2}}{2 m} \frac{\nabla^{2} R}{R}-V(R)=E
$$

and

$$
\frac{S \nabla^{2} S}{m}-\frac{(\nabla S)^{2}}{2 m}=E .
$$

Equation (8) can be expressed as

$$
-\frac{\hbar^{2}}{2 m} \nabla^{2} R+V R=-E R,
$$

which is the Schrödinger equation for a particle of mass $m$ and total energy $-E$ whose wave function is $R$. Thus, unlike the Bohmian formulation, where $R$ doesn't satisfy the Schrodinger's equation, the function $R$ in our present formulation satisfies the Schrodinger's equation. Equation (9) is a non linear differential equation in 
the variable $S$ which can be solved to give the spatial dependance of $S$. The solution of eq.(9) in one dimension is

$$
S=\frac{2 m E}{C}+\frac{C}{4} x^{2}
$$

where $C$ is a constant having a dimension of $\mathrm{kg} / \mathrm{s}$.

Using the definition $\vec{p}=\vec{\nabla} S$, eq. $(9)$ can be expressed as

$$
\frac{p^{2}}{2 m}-\frac{S \nabla^{2} S}{m}=-E .
$$

One can define the term

$$
V_{S}=-\frac{S \nabla^{2} S}{m}
$$

as a spin potential. This can be juxtaposed with the quantum potential, $Q$, that depends on $\hbar^{2}$. This potential arises from an internal motion of the particle. Like the quantum potential, that is proportional to the curvature due to $R$, the spin potential is proportional to the curvature due to $S$. Applying eq.(a) in eq.(12) yields $V_{S}=-E-\left(C^{2} / 8 m\right) x^{2}$. This gives rise to a conservative force, $F=-\partial V_{S} / \partial x=\left(C^{2} / 4 m\right) x$. Such a potential is found to governed the nucleons (proton and neutron) inside a nucleus.

While the quantum potential is scale invariant $(R \rightarrow \beta R$, for some constant $\beta)$, the spin potential is not and scales quadratically. That is because the amplitude $R$ can always be normalized to unity, but $S$ is not. Moreover, while the quantum potential can be singular, the spin potential is always regular and definite.

The spin force associated with this potential is given by

$$
F_{S}=-\vec{\nabla} V_{S}=S \frac{\nabla^{2}(\vec{\nabla} S)}{m}+\frac{(\vec{\nabla} S)}{m} \nabla^{2} S,
$$

or

$$
F_{S}=S \nabla^{2} \vec{v}+\left(\nabla^{2} S\right) \vec{v}
$$

where $\vec{v}=\frac{\vec{\nabla} S}{m}$. Since the second term in the force $F_{S}$ depends on the particle velocity, it can be seen as a drag force. The first term accounts for the viscosity force that is similar to the term appearing in the Navier-Stokes equation. It may usher in a direction of an existence of a permeating fluid filling the whole space that were once proposed for Maxwell's waves (the ether). The second term can be expressed as $\nabla^{2} S=\vec{\nabla} \cdot(\vec{\nabla} S)$ which can be written as $m \vec{\nabla} \cdot \vec{v}$. Hence, the spin force in eq.(13) will read

$$
F_{S}=S \nabla^{2} \vec{v}+m \vec{v}(\vec{\nabla} \cdot \vec{v})
$$

Thus, because of its spin, the quantum particle would appear to be moving in a viscous fluid. The first term in eq.(14) suggests that the spin of the electron is a measure of some intrinsic coefficient of viscosity, since the 
viscous force density is proportional to $\eta \nabla^{2} \vec{v}$, where $\eta$ is the coefficient of viscosity. Hence, $\eta$ represents the spin density. While the drag force depends on the particle mass (inertia), the viscosity force does not. This amounts to say that it is associated with some internal degree of freedom that the particle has. And since, the electron spin $S \neq 0$, the viscous force can never vanish. It is shown by Schrodinger that the free electron, in Dirac's theory, exhibits a jittery motion. This motion may be connected with this force.

It is found recently that the Hall's viscosity is proportional to the average spin [5]. In the realm of particle physics, hadrons consist of three quarks that move freely inside them. Consequently, one may argue that a similar situation exits inside the electron.

For an incompressible fluid, $\vec{\nabla} \cdot \vec{v}=0$, and therefore, $F_{S}=S \nabla^{2} \vec{v}$, which implies that the spin force is independent of the particle mass.

Let us digress a bit and look at eq.(11) as defining the energy conservation of a free particle $(V(R)=0)$ being influenced by its internal motion only, viz.,

$$
-E=\frac{p^{2}}{2 m}+V_{S}
$$

upon using eq.(12). Using the vector identity

$$
\vec{\nabla} \cdot(S \vec{\nabla} S)=(\nabla S)^{2}+S \nabla^{2} S
$$

eq.(9) can be expressed as

$$
\frac{3 p^{2}}{2 m}+E=\vec{\nabla} \cdot\left(S \frac{\vec{\nabla} S}{m}\right),
$$

or

$$
\frac{3 p^{2}}{2 m}+E=\vec{\nabla} \cdot(S \vec{v})
$$

However, if $S$ is conserved, then

$$
\frac{d S}{d t}=\frac{\partial S}{\partial t}+\vec{\nabla} \cdot(S \vec{v})=0
$$

so that eq.(18) can be written as

$$
\frac{\partial S}{\partial t}+\left(\frac{3 p^{2}}{2 m}+E\right)=0
$$

The factor 3 in the above equation may be attributed to rotation of the particle in 3-dimensions. Equation (20) can be expressed in the Hamilton-Jacobi format to read

$$
\frac{\partial S}{\partial t}+H_{p}=0
$$

where

$$
H_{p}=\frac{p^{2}}{2 m}+\frac{p^{2}}{m}+E
$$


is the Hamiltonian of the system. The extra kinetic energy term, $\frac{p^{2}}{m}$, may be attributed to spin kinetic energy contributed by the two subfields $(R$ and $S)$ comprising the quantum particle.

Canonical momenta. - Let us express the momentum related to $S$ as $\vec{p}_{S}=\vec{\nabla} S$ and that is related to $R$ as $\vec{p}_{R}=\hbar \vec{\nabla} R$. Equations (1) and (2) can be expressed as

$$
\frac{\partial S}{\partial t}+H_{S}=0, \quad \frac{\partial \mathcal{R}}{\partial t}+H_{R}=0
$$

where

$$
H_{S}=\frac{(\nabla S)^{2}}{2 m}+V-\frac{\hbar^{2}}{2 m} \frac{\nabla^{2} R}{R}
$$

and

$$
\left.H_{R}=\frac{\hbar}{2 m}\left(R \nabla^{2} S+2 \vec{\nabla} R \cdot \vec{\nabla} S\right)\right)
$$

are the Hamiltonians associating with the two actions, $S$ and $\mathcal{R}=\hbar R$, respectively.

Thus, one can use $R$ and $S$ as generalized coordinates and $p_{R}$ and $p_{S}$ as their corresponding generalized momenta. To make $R$ have a dimension of length and $S$ have a dimension of momentum, we multiple $R$ by a constant $\lambda$ and divide $S$ by a constant $p_{\lambda}$ having dimensions of length and momentum, respectively, viz., $\tilde{S}=\frac{S}{p_{\lambda}}$ and $\tilde{R}=\lambda R$.

In terms of the momenta $p_{R}$ and $p_{S}$, eqs.(24) and (25) can be written as

$$
H_{S}=\frac{p_{S}^{2}}{2 m}+V-\frac{\hbar \lambda}{2 m \tilde{R}} \vec{\nabla} \cdot \overrightarrow{p_{R}}
$$

and

$$
H_{R}=\frac{\hbar \tilde{R}}{2 m \lambda \tilde{S}} \vec{\nabla} \cdot\left(\tilde{S} \vec{p}_{S}\right)-\frac{\hbar \tilde{R} p_{S}^{2}}{2 m \lambda p_{\lambda} \tilde{S}}+\frac{\vec{p}_{R} \cdot \vec{p}_{S}}{m} .
$$

In this case, the Hamilton's equations become

$$
\dot{\tilde{R}}=\frac{\partial H_{R}}{\partial p_{R}}, \quad \dot{p}_{R}=-\frac{\partial H_{R}}{\partial \tilde{R}}
$$

and

$$
\dot{\tilde{S}}=\frac{\partial H_{S}}{\partial p_{S}}, \quad \dot{p}_{S}=-\frac{\partial H_{S}}{\partial \tilde{S}},
$$

to solve for $\tilde{R}$ and $\tilde{S}$. Apply eq.(28) in eq.(27) to obtain

$$
\dot{R}=\frac{p_{S}}{\lambda m}, \quad \dot{p}_{R}=\frac{\hbar p_{S}^{2}}{2 m S \lambda}-\frac{\hbar}{2 m S \lambda} \vec{\nabla} \cdot\left(S \vec{p}_{S}\right),
$$

and apply eq.(29) in eq.(26) to obtain

$$
\dot{S}=\frac{p_{\lambda} p_{S}}{m}, \quad \dot{p}_{S}=-p_{\lambda} \frac{\partial V}{\partial S}
$$


An educated guess for the relation between $\lambda$ and $p_{\lambda}$ is that $\lambda=h / p_{\lambda}$, where $h$ is the Planck's constant. If we assume that $V$ is a function of $R$ only, then $\dot{p}_{S}=0$. Hence, $R$ and $S$ are constants too. This yields the relation $S=h R$. This is a very interesting relation relating the wave amplitude to its phase (spin). In this particular case, $V_{S}=V_{Q}$.

Recall that writing the wavefunction in the form $\psi=R e^{i S / \hbar}$ may be seen as defining the point in a complex plane, viz., $z=r e^{i \theta}$, where $r^{2}=|z|^{2}$ and $\theta$ is the angle that $r$ subtends with the $\mathrm{x}$-axis. In this case, the relation $S=h R$ implies that $S / \hbar=2 \pi R$ is the circumference of the circle with radius $R$. Winding the circle $n$ times, for some integer $n$, doesn't change the phase (we come to the same point we started with). Hence, we can say that $S=n \hbar$ for constant $R$.

Spin dependent potential. - Let us now assume that $V$ depends on $S$. In this case eq.(7) reads

$$
\frac{\hbar^{2}}{2 m} \frac{\nabla^{2} R}{R}=-E_{S}
$$

and

$$
-E_{S}=\frac{S \nabla^{2} S}{m}-\frac{(\nabla S)^{2}}{2 m}+V(S)
$$

Equations (32) and (33) can be written as

$$
\nabla^{2} R+\frac{2 m E_{S}}{\hbar^{2}} R=0
$$

and

$$
E_{S}=\frac{p_{S}^{2}}{2 m}+V_{T}, \quad V_{T}=-\left(V+\frac{S \nabla^{2} S}{m}\right),
$$

where $V_{T}$ is the total potential of the system. Therefore, the amplitude $R$ satisfies the Schrödinger equation for a particle with total energy equals to $E_{S}$.

Bohmian Dirac's equation. - Let us now explore the Dirac's equation (where $\vec{\alpha}$ and $\beta$ are $4 \times 4$ matrices)

$$
i \hbar \frac{\partial \psi}{\partial t}=-i \hbar c \vec{\alpha} \cdot \vec{\nabla} \psi+\beta m c^{2} \psi
$$

by writing the wavefunction $\psi$ as $\psi=R e^{i S / \hbar}$, where $S$ and $R$ can be seen as 4-components real functions. Equating the real and imaginary parts in the two sides of the resulting equation yield, respectively,

$$
\frac{\partial S}{\partial t}+\left(c \vec{\alpha} \cdot \vec{\nabla} S+\beta m c^{2}\right)=0, \quad \frac{\partial R}{\partial t}+c \vec{\alpha} \cdot \vec{\nabla} R=0
$$

Since the velocity in Dirac formulation is given by $\vec{v}=c \vec{\alpha}$, then eq.(37) will be

$$
\left(\frac{\partial S}{\partial t}+\vec{v} \cdot \vec{\nabla} S\right)+\beta m c^{2}=0, \quad \frac{\partial R}{\partial t}+\vec{v} \cdot \vec{\nabla} R=0
$$


Equation (38) can be written as

$$
\frac{d S}{d t}+\beta m c^{2}=0, \quad \frac{d R}{d t}=0
$$

or by using the relation $\vec{v}=\frac{\vec{\nabla} S}{m}$ in eq.(38) yields

$$
\frac{\partial S}{\partial t}+\left(\frac{(\nabla S)^{2}}{m}+\beta m c^{2}\right)=0, \quad \frac{\partial R}{\partial t}+\frac{\vec{\nabla} S \cdot \vec{\nabla} R}{m}=0 .
$$

The second equation in eq.(39) implies that the probability (amplitude) is time independent. The first equation in eq.(40) suggests that the Dirac total energy in the classical world is given by $E_{D}=\frac{p^{2}}{m} \pm m c^{2}$.

Equation (40) can be seen as representing two Hamilton-Jacobi equation with actions $S$ and $\mathcal{R}=\hbar R, v i z$.

$$
\frac{\partial S}{\partial t}+H_{S}=0, \quad H_{S}=\frac{(\nabla S)^{2}}{m}+\beta m c^{2},
$$

and

$$
\frac{\partial \mathcal{R}}{\partial t}+H_{R}=0, \quad H_{R}=\frac{\hbar}{m} \vec{\nabla} S \cdot \vec{\nabla} R
$$

The two Hamiltonians in eq.(41) and (42) can be expressed as

$$
H_{S}=\frac{p_{S}^{2}}{m}+\beta m c^{2}, \quad H_{R}=\frac{\vec{p}_{S} \cdot \vec{p}_{R}}{m},
$$

so that Hamilton's equations yield

$$
\frac{d R}{d t}=\frac{p_{S}}{\lambda m}, \quad \frac{d S}{d t}=\frac{2 p_{S} p_{\lambda}}{m}
$$

where $p_{S}=$ const., since $H_{S}$ is independent of $S$, and $H_{R}$ is independent of $R$, respectively.

Bohmian Klein-Gordon equation. - Let us now consider the Klein-Gordon equation

$$
\frac{1}{c^{2}} \frac{\partial^{2} \phi}{\partial t^{2}}-\nabla^{2} \phi+\left(\frac{m c}{\hbar}\right)^{2} \phi=0
$$

and substitute instead $\phi=R e^{i S / \hbar}$. Separating the real and imaginary parts in the resulting equation, yields

$$
\frac{1}{c^{2}} \ddot{R}-R^{\prime \prime}+\left(\frac{m^{2} c^{2}}{\hbar^{2}}+\frac{S^{\prime 2}}{\hbar^{2}}-\frac{\dot{S}^{2}}{c^{2} \hbar^{2}}\right) R=0,
$$

and

$$
\frac{1}{c^{2}} \ddot{S}-S^{\prime \prime}+2\left(\frac{1}{c^{2}} \frac{\dot{R}}{R} \frac{\dot{S}}{S}-\frac{R^{\prime}}{R} \frac{S^{\prime}}{S}\right) S=0 .
$$

If we allow $R$ to satisfy the Klein-Gordon equation, then $c S^{\prime}=\dot{S}$. This latter equation implies that $S$ satisfies a wave traveling at the speed of light. Conversely, if we allow $R$ to satisfy the wave equation traveling at the speed of light, then eq.(46) would imply that $S$ satisfies the equation, $\dot{S}^{2}=c^{2} S^{\prime 2}+m^{2} c^{4}$, which upon using the Hamilton-Jacobi equation, $E=-\frac{\partial S}{\partial t}=-\dot{S}$ and $\vec{p}=\vec{\nabla} S=S^{\prime}$ yields $E^{2}=c^{2} p^{2}+m^{2} c^{4}$ that is the Einstein 
relativistic energy-momentum equation. A massless particle satisfies the two wave equations $c R^{\prime}=\dot{R}$ and $c S^{\prime}=\dot{S}$. Interestingly, the amplitude and the phase of the wavefunction of a massless particle satisfy the wave equation traveling at the speed of light.

Equations (46) and (47) suggest that the particle consists of two subparticles whose fields satisfy the KleinGordon equation with masses

$$
M_{R}^{2}=m^{2}+\frac{S^{\prime 2}}{c^{2}}-\frac{\dot{S}^{2}}{c^{4}}, \quad M_{S}^{2}=\frac{2 \hbar^{2}}{c^{2}}\left(\frac{1}{c^{2}} \frac{\dot{R} \dot{S}}{R S}-\frac{R^{\prime} S^{\prime}}{R S}\right) .
$$

The mass of the $R$ field depends solely on $S$ fields, but that due to $S$ field depends on $S$ and $R$ fields and their spatial and temporal derivatives. Hence, the mass of each subfield depends on the interaction between these subfields. Thus, the field $\phi$ is composed of these two subfields (eigen functions). This is manifested in writing $\phi$ as $\phi=U+i W$, where $U=R \cos (S / \hbar)$ and $W=R \sin (S / \hbar)$, where $U$ and $W$ satisfy the Klein-Gordon equation.

Bohmian Unified quantum wave equation. - Let us now apply the wavefunction representation, $\psi_{0}=R \exp (i S / \hbar)$, in the unified quantum wave equation [4]

$$
\frac{1}{c^{2}} \frac{\partial^{2} \psi_{0}}{\partial t^{2}}-\nabla^{2} \psi_{0}+\frac{2 m}{\hbar} \frac{\partial \psi_{0}}{\partial t}+\left(\frac{m c}{\hbar}\right)^{2} \psi_{0}=0 .
$$

Equating the real and imaginary parts in the two sides of the resulting equation to each other, yields

$$
\frac{\ddot{R}}{c^{2}}-R^{\prime \prime}+\frac{2 m}{\hbar} \dot{R}+\left(\frac{m^{2} c^{2}}{\hbar^{2}}-\frac{\dot{S}^{2}}{c^{2} \hbar^{2}}+\frac{S^{\prime 2}}{\hbar^{2}}\right) R=0
$$

and

$$
\frac{\ddot{S}}{c^{2}}-S^{\prime \prime}+\frac{2 m}{\hbar} \dot{S}+2\left(\frac{1}{c^{2}} \frac{\dot{R}}{R} \frac{\dot{S}}{S}-\frac{R^{\prime}}{R} \frac{S^{\prime}}{S}\right) S=0
$$

where the dot denotes the partial derivative with respect to time and the prime denotes the partial derivative with respect to space.

It is apparent that if we assume $R$ satisfy eq.(49), then one finds $\dot{S}=c S^{\prime}$. This implies that $S$ satisfies a wave equation with zero mass, viz., $\frac{\ddot{S}}{c^{2}}-S^{\prime \prime}=0$, while $R$ satisfies a wave equation with non-zero mass. Thus, the phase information travels at the speed of light. Therefore, the wavefunction $\psi_{0}$ contains two kinds of waves. Hence, particles and waves are hybrid (coexist). The two waves are coupled as evident from eqs.(50) and (51). However, if we assume that $R$ satisfy a modulated Klein-Gordon equation (telegraph equation), i.e.,

$$
\frac{\ddot{R}}{c^{2}}-R^{\prime \prime}+\frac{2 m}{\hbar} \dot{R}=0
$$

then eq.(50) yields

$$
\frac{\dot{S}^{2}}{m^{2} c^{4}}-\frac{S^{2}}{m^{2} c^{2}}=1
$$


which is an equation of a hyperbola with coordinates, $\dot{S}$ and $S^{\prime}$. Thus, the phase evolves dynamically and at any given time is controlled by the hyperbola equation.

Equations (50) and (51) indicate that in the new functions, $S$ and $R$, the quantum particle appears to be consisting of two interacting subparticles satisfying eq.(49). The masses of these subparticles are, respectively,

$$
M_{R}^{2}=m^{2}-\frac{\dot{S}^{2}}{c^{4}}+\frac{S^{\prime 2}}{c^{2}}, \quad M_{S}^{2}=\frac{2 \hbar^{2}}{c^{4}} \frac{\dot{R}}{R} \frac{\dot{S}}{S}-\frac{2 \hbar^{2}}{c^{2}} \frac{R^{\prime}}{R} \frac{S^{\prime}}{S}
$$

It is important to compare this formulation with Schrödinger one. In Schrödinger formulation, the wave amplitude $R$ is not coupled with the phase, $S$. Moreover, $S$ satisfies the quantum Hamilton-Jacobi equation but $R$ doesn't satisfy Schrödinger equation. In the present formulation while $R$ is a decaying oscillatory function of time satisfying the telegraph equation, the phase satisfies a wave equation traveling at the speed of light. Hence, the phase information is dispatched everywhere at the speed of light. Moreover, the field $\psi_{0}$ is composed of two subfields, $U$ and $W$, such that $\psi_{0}=U+i W$, where $U=R \cos (S / \hbar)$ and $W=R \sin (S / \hbar)$. Note that $U$ and $W$ satisfy the wave equation in eq. $(49)$

Let us now consider the special case, when $\dot{R}=0$ and $\dot{S}=0$ and substitute them in eqs.(50) and (51) to obtain

$$
\hbar^{2} c^{2} \frac{\nabla^{2} R}{R}=m^{2} c^{4}+c^{2} S^{2}, \quad \quad S^{\prime}=A R^{-2}, \quad A=\text { const. }
$$

Hence, the quantum potential defined in Schrodinger case can be expressed by

$$
V_{Q}=-\frac{\hbar^{2}}{2 m} \frac{\nabla^{2} R}{R}=-\frac{m c^{2}}{2}-\frac{A^{2}}{2 m R^{4}}
$$

which is an attractive potential. Since as $r \rightarrow \infty$, the probability, $R^{2} \rightarrow 0$, the quantum potential $V_{Q} \rightarrow-\infty$. This indicates that the influence of the quantum potential (force) is present even at large scale. The quantum potential may be responsible for the splitting (creation/annihilation) of the $\psi_{0}$ field into $U$ and $W$ fields each having a mass of $m c^{2}$. These two fields oscillate internally so as to endow the particle its quantum effects (spin, jittery motion, etc.).

Concluding remarks. - We have revisited in this paper the Bohmian quantum mechanics by considering that the continuity equation can be set to represent an additional Hamilton-Jacobi equation; and solve the two Hamilton equations treating $S$ and $R$ as two generalized co-ordinates. In doing so, a new potential, called the spin potential is obtained, that is proportional to the curvature of $S$. This is a classical potential that doesn't incorporate the Planck's constant. The expectation value of the spin density is found to be conserved. We then related this expectation value to the classical spin of the particle. The spin potential gives rise to a viscosity force whose coefficient of viscosity is directly proportional to the spin. Furthermore, we apply the Bohmian recipe of quantum mechanics to the unified quantum wave equation that we have recently derived. Besides, we have 
shown that if $R$ satisfies the telegraphic de Broglie equation (undistorted telegraph equation), then $S$ satisfies the wave equation traveling at the speed of light. However, if we assume $R$ satisfy the telegraph equation, then $S$ satisfies the Einstein relativistic energy-momentum equation expressed in terms of the momentum $\vec{p}=\vec{\nabla} S$ and energy $E=-\frac{\partial S}{\partial t}$. Applying the Bohmian recipe to the Dirac's equation shows that $R^{2}$ is conserved and the rate of change of the spin density, $\frac{d}{d t}\left(R^{2} S\right)=-\beta m c^{2} R^{2}$.

Furthermore, it is shown that the functions $R$ and $S$ represent two subfields from which the quantum particle is formed, and the interaction between them provides their masses.

\section{REFERENCES}

[1] D. Bohm, A suggested interpretation of quantum mechanics theory in terms of "hidden" variables, I, Phys. Rev. 85, 166, 1952.

[2] D. Bohm, A Suggested Interpretation of the Quantum Theory in Terms of "Hidden" Variables, II, Phys. Rev. 85, $180,1952$.

[3] de Broglie, L., La mécanique ondulatoire et la structure atomique de la matière et du rayonnement, J. Phys. Radium 8, 225, 1927.

[4] A. I. Arbab, Derivation of Dirac, Klein-Gordon, Schrödinger, Diffusion and quantum heat transport equations from a universal quantum wave equation, EPL, 92, 40001, 2010.

[5] N. Read, Non-Aeolian adiabatic statistics and Hall viscosity in quantum Hall states and $p_{x}+$ ip $p_{y}$ paired superfluids, Phys. Rev. B79, 045308, 2009. 